\title{
The Obv-Eas Method: An Easy Way to Facilitate Fiberoptic Intubation in Pediatric Patients: Case of an Infant with Freeman-Sheldon Syndrome
}

\author{
Ozkan Onal ${ }^{1,{ }^{*}}$, Irem Gumus ${ }^{2}$, Mehmet Sari ${ }^{2}$, Muhammed Emin Zora ${ }^{2}$ and Mehmet Ali Acar ${ }^{2}$ \\ ${ }^{1}$ Anesthesiology and Reanimation, Selcuk University Medical Faculty, Konya, Turkey \\ ${ }^{2}$ Orthopedics Surgery, Selcuk University Medical Faculty, Konya, Turkey \\ "Corresponding author: Selcuk University Medical Faculty, Konya, Turkey. Tel: +90-5059052252, E-mail: drozkanonal@selcuk.edu.tr
}

Received 2018 June 29; Revised 2018 August 29; Accepted 2018 September 08.

Keywords: Freeman-Sheldon Syndrome, Intubation, Intratracheal, Investigative Techniques, Bronchoscope, Airway Management, Infant

\section{Dear Editor,}

We describe a case of Freeman-Sheldon syndrome(FSS) in a 22-month-old infant weighing $6.5 \mathrm{~kg}$ scheduled to undergo knee web-plasty in the prone position, in which fiber-optic intubation was successfully performed in an easy way. We briefly review the features of FSS, discuss anesthetic challenges associated with intubation of pediatric patients, and describe the ease and success of fiber-optic intubation using a new technique.

FSS, also known as cranio-carpo-tarsal dysplasia and whistling face syndrome, is a rare congenital myopathy disorder first reported in 1938 and characterized by malformations of the face, oral cavity, and musculoskeletal system (1). Common facial features include microstomia, microglossia, a high arched palate, hypoplastic mandible, and a short neck with relatively limited mobility. The combination of abnormal facial characteristics makes direct laryngoscopy and orotracheal intubation difficult or impossible (2). According to the literature, the most frequent challenge during anesthesia is difficult intubation. Studies also reported that FSS can be related with muscle rigidity, pyrexia, and malignant hyperthermia, however, these findings have not been confirmed $(3,4)$. Our patient was a 22month-old with Freeman-Sheldon syndrome (FSS) scheduled for web-plasty in the prone position. The male patient weighed only $6.5 \mathrm{~kg}$ and had dysmorphic features (Figure 1), with retrognathia, microstomia, microglossia, a high arched palate, a hypoplastic mandible, ulnar deviation of fingers, bilateral congenital talipes equinovarus, and knee dislocation. Intubation had been unsuccessful on two previous occasions due to the presence of the afore- mentioned anomalies associated with FSS. Given the patient's history of unsuccessful intubation and the need to perform the surgery in the prone position, we chose fiberoptic-assisted nasotracheal intubation, with the patient sedated but maintaining spontaneous respiration. In addition, spinal anesthesia was administered to prevent postoperative pain. The procedure was explained to the parents and they provided signed informed consent for a combination of regional and general anesthesia. Preoperative chest radiography, electrocardiography (ECG), hematological and biochemical investigations, baseline vitals recorded using ECG, pulse oximetry, and noninvasive blood pressure were normal. A skin temperature probe was placed after intubation. Anesthesia was induced via a mask with sevoflurane, oxygen, and nitrous oxide, with gradual increases in concentration every few breaths, up to a concentration of $6 \%$. Upon loss of consciousness, the sevoflurane-inspired concentration was reduced to $2 \%$ $4 \%$. Anesthesia was achieved via incremental doses of 1 $\mathrm{mg} / \mathrm{kg}$ propofol (Lipuro, Braun, Germany) administered intravenously. Spontaneous respiration was maintained during the induction of intravenous (i.v.) anesthesia.

Atropine $0.2 \mathrm{mg}$ was administered intravenous due to mild bradycardia (HR: 70 beat/ min.) and to dry copious oral secretions. As the epiglottis was not visible by direct laryngoscopy and the patient had a history of difficult intubation, we decided to proceed with fiberoptic intubation using a new, OBVIOUS, and EASY technique we developed (obv-eas) as described below.

After the application of Ksilometazoline Hcl (Otrivine, Novartıs, Turkey) into both nares, an uncuffed tracheal 

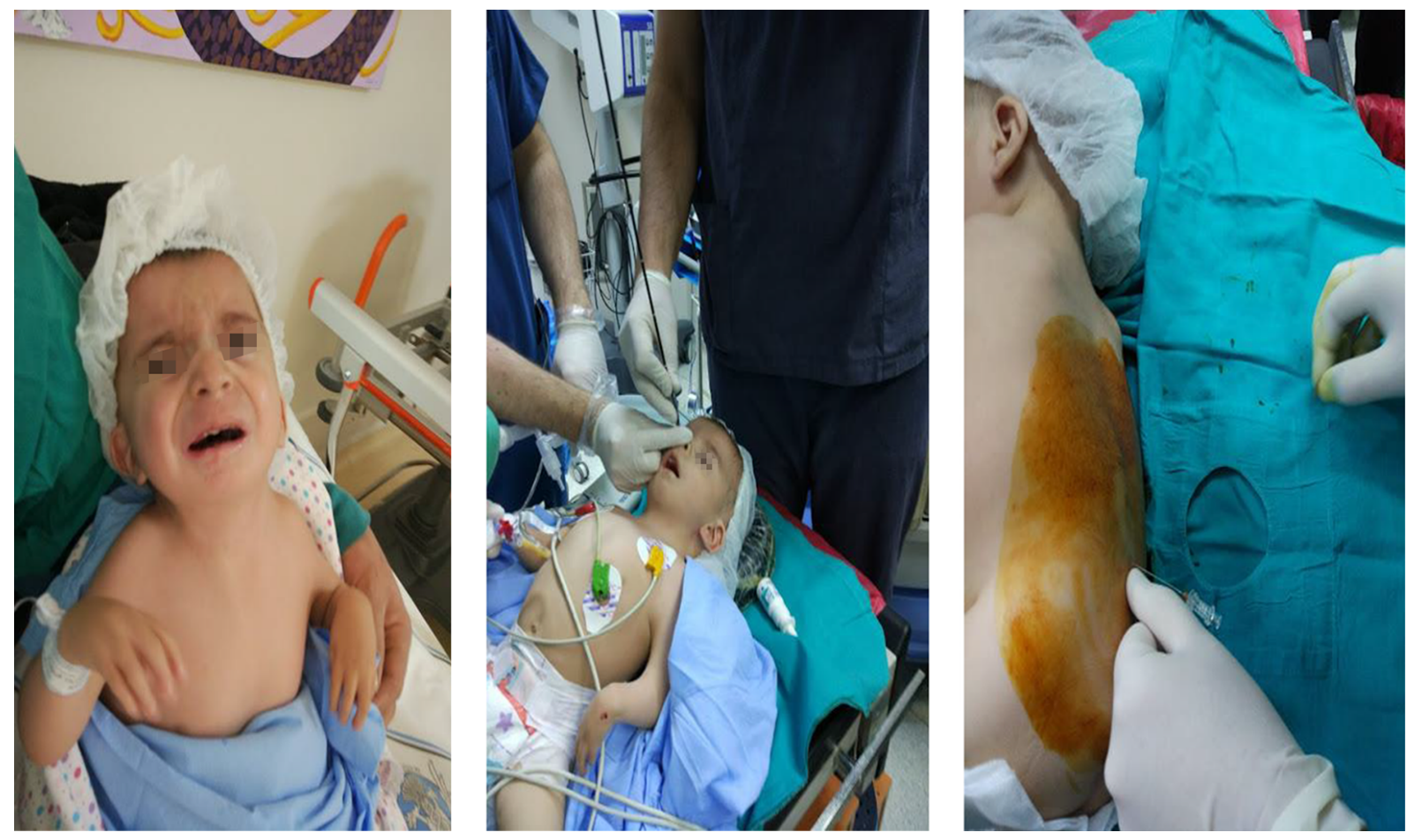

Figure 1. The image of the patient before surgery, during fiberoptic intubation, and during spinal anesthesia administration respectively.

tube with an internal diameter of $4.0 \mathrm{~mm}$ (Kendall- Gammatron, USA) was lubricated and inserted into the right nares until it reached a point close to the hypopharynx. A fiberoptic bronchoscope (Karl Storz, Germany) with an outer diameter (OD) of $3.7 \mathrm{~mm}$ was then inserted through the tube lumen. In this way, we prevented the breakdown of fiberoptic and the release of secretions that would reduce our vision. Meanwhile, we maintained oxygen by flushing via the patient's mouth. The oxygen saturation did not fall below $100 \%$. The nasal fiberoptic bronchoscope entered the trachea easily and the tracheal tube was advanced over the bronchoscope and positioned about $1 \mathrm{~cm}$ above the carina (Figure 1). Anesthesia induction was completed using $3 \mathrm{mg}$ of i.v. rocuronium (Esmeron, Organon, Turkey) and fentanyl $10 \mathrm{mcg}$ (Talinat, Vem, Turkey). Anesthesia was maintained using $70 \%$ nitrous oxide in a 30\% oxygen and propofol (Propofol, Fresenius, Turkey) infusion (0.125 mg/kg).

To reduce postoperative pain and make the joints loose during the surgery, we performed spinal anesthesia. The patient was carefully positioned in the right lateral kneechest position. Then, $1 \mathrm{mg} / \mathrm{kg}$ of $0.5 \%$ bupivacaine (Marcaine, Eczacibasi, Turkey) was administered intrathecally using a 26G Quincke (Atraucan, Braun, Germany) spinal needle at the level of L4-L5 and rate of $0.1 \mathrm{~mL} / \mathrm{sec}$. The needle was withdrawn after maintaining it in this position for $5 \mathrm{sec}$ (Figure 1). Surgery was completed uneventfully. The patient did not exhibit any signs of malignant hyperthermia during or after the procedure, and the trachea was extubated in the operating room. Intubation may be difficult for a number of reasons in pediatric patients, including those with FSS. The conventional nasal fiberoptic intubation technique may be impractical in some patients, especially in very young pediatric patients due to FOB refraction and secretion. Rapid desaturation is an additional problem in pediatric patients. The proposed nasal fiberoptic intubation technique is easy and should be considered an option in difficult intubation cases involving pediatric patients. We have intubated an infant patient who hasn't been intubuated twice before with our newly defined technique by using fiberoptic. Our method differs from the others in that the fiberoptic is sent to the blindfold through the intubation tube to prevent the damage of the fiberoptic and to prevent the deterioration of the fiberoptic view due to the secretions.

There are a few publications in the literature on the management of anesthesia in patients with FSS syndrome $(1,3-6)$. In some case reports, inhalation anesthesia was used $(1,3,5,6)$ whereas in some case reports tiva was used (5). We have also used inhalation anesthesia due to the 
fact that the use of inhalation anesthesia is not strictly contraindicated in this syndrome and additionally there have been case reports using inhalation anesthesia in the literature $(1,3,5,6)$.

\section{References}

1. Kim JS, Park SY, Min SK, Kim JH, Lee SY, Moon BK. Awake nasotracheal intubation using fiberoptic bronchoscope in a pediatric patient with Freeman-Sheldon syndrome. Paediatr Anaesth. 2005;15(9):790-2. doi: 10.1111/j.1460-9592.2004.01533.x. [PubMed: 16101713].

2. Stevenson DA, Carey JC, Palumbos J, Rutherford A, Dolcourt J, Bamshad MJ. Clinical characteristics and natural history of
Freeman-Sheldon syndrome. Pediatrics. 2006;117(3):754-62. doi: 10.1542/peds.2005-1219. [PubMed: 16510655].

3. Jones R, Dolcourt JL. Muscle rigidity following halothane anesthesia in two patients with Freeman-Sheldon syndrome. Anesthesiology. 1992;77(3):599-600. [PubMed: 1519800].

4. Laishley RS, Roy WL. Freeman-Sheldon syndrome: Report of three cases and the anaesthetic implications. Can Anaesth Soc J.1986;33(3 Pt 1):388-93. [PubMed: 3719442].

5. Fiorelli S, Leopizzi G, Massullo D, D’Andrilli A, Ciccone AM, Rocco M. Anesthetic management of a patient with Freeman-Sheldon syndrome in thoracic surgery. J Clin Anesth. 2018;48:48-9. doi: 10.1016/j.jclinane.2018.05.008. [PubMed: 29751210].

6. Hamilton T, Sathyamoorthy M. A case of Freeman-Sheldon syndrome: Anesthetic challenges. J Miss State Med Assoc. 2016;57(1):6-8. [PubMed: 27111982]. 